\title{
Linear Energy Transfer-Optimized Intensity Modulated Proton Therapy
}

National Cancer Institute

\section{Source}

National Cancer Institute. Linear Energy Transfer-Optimized Intensity Modulated Proton

Therapy. NCI Thesaurus. Code C155822.

Techniques for maximizing LET in targ et volumes and minimizing LET in critical structures and normal tissues during proton beam radiation therapy. 